\title{
A Wire-Based Active Tracker
}

\author{
Juan Andrade-Cetto, Member, IEEE, and Federico Thomas, Member, IEEE
}

\begin{abstract}
Wire-based tracking devices are an affordable alternative to costly tracking devices. They consist of a fixed base and a platform, attached to the moving object, connected by six wires whose tension is maintained along the tracked trajectory. One important shortcoming of these devices is that they are forced to operate in reduced workspaces so as to avoid singular configurations. Singularities can be eliminated by adding more wires, but this causes more wire interferences, and a higher force exerted on the moving object by the measuring device itself. This paper shows how, by introducing a rotating base, the number of wires can be reduced to three, and singularities can be avoided by using an active sensing strategy. This also permits reducing wire interference problems and the pulling force exerted by the device.
\end{abstract}

Index Terms-Active sensing, Kalman filtering, mutual information, parallel manipulators, tracking devices.

\section{INTRODUCTION}

$\mathbf{T}$ RACKING devices are used for estimating the position and orientation of moving objects, and are often based on electromagnetic, acoustic, mechanical, or optical technology. Tracking devices can be classified according to their characteristics, such as accuracy, resolution, cost, measurement range, portability, and calibration requirements. Laser tracking systems exhibit good accuracy, which can be less than $1 \mu \mathrm{m}$ if the system is well calibrated. Unfortunately, this kind of systems are very expensive, their calibration procedure is time consuming, and they are sensitive to the environment. Vision systems can reach an accuracy of $0.1 \mathrm{~mm}$. They are low-cost portable devices but their calibration procedure can be complicated. Wire-based systems can reach an accuracy of $0.1 \mathrm{~mm}$, they are also low-cost portable devices but capable of measuring large displacements. Moreover, they exhibit a good compromise among accuracy, measurement range, cost and operability.

Wire-based tracking devices consist of a fixed base and a platform connected by six wires whose tension is maintained, while the platform is moved, by pulleys and spiral springs on the base, where a set of encoders give the length of the wires. They can be modeled as 6-DOF parallel manipulators because wires can be seen as extensible legs connecting the platform and the base by

Manuscript received September 7, 2007; revised February 22, 2008. This paper was recommended for publication by Associate Editor I. Bonev and Editor $\mathrm{K}$. Lynch upon evaluation of the reviewers' comments. This work was supported in part by the European Union (EU) under project Perception, Action, and Cognition through Learning of Object-Action-Complexes (PACO-PLUS) project under Grant FP6-2004-IST-4-27657, in part by the Consolider Ingenio 2010 project under Grant CSD2007-00018, and in part by the Spanish Ministry of Education and Science project under Grant DPI 2007-60858. The work of J. Andrade-Cetto was supported by the Spanish Ministry of Education and Science under the Ramón y Cajal Postdoctoral Fellowship.

The authors are with the Spanish National Research Council, Institut de Robòtica i Informàtica Industrial, 08028 Barcelona, Spain (e-mail: cetto@ iri.upc.edu; fthomas@ iri.upc.edu).

Color versions of one or more of the figures in this paper are available online at http://ieeexplore.iee.org.

Digital Object Identifier 10.1109/TRO.2008.924260 means of spherical and universal joints, respectively. Some sample applications of wire-based tracking include haptic devices for virtual reality systems for the visually impaired [1] or for the characterization of singularities of parallel mechanisms [2].

Dimension deviations due to fabrication tolerances, wirelength uncertainties, or wire slackness, may result in unacceptable performance of a wire-based tracking device. In general, the effects of all systematic errors can be eliminated by calibration. Some techniques to calibrate for specific errors have already been proposed in the literature. For example, a method for compensating the cable guide outlet shape of wire encoders is detailed in [3], and a method for compensating the deflections caused by wire self-weights is described in [4]. In this paper, we will only consider wire-length errors that cannot be compensated because of their random nature.

Another indirect source of error is the force exerted by the measuring device itself. Indeed, all commercial wire encoders are designed to keep a large string tension. This is necessary to ensure that the inertia of the mechanism does not result in a wire going slack during a rapid motion. If a low wire force is used, it would reduce the maximum speed of the object to be tracked without the wires going slack. On the contrary, if a high wire force is used, the trajectory of the object to be tracked could be altered by the measuring device. Hence, a tradeoff between accuracy and speed arises.

The minimum number of points on a moving object to be tracked for pose measurements is three. Moreover, the maximum number of wires attached to a point is also three; otherwise the lengths of the wires would not be independent. This leads to only two possible configurations for the attachments on the moving object: the 3-2-1 configuration and the 2-2-2 configuration. The application of the 3-2-1 configuration to pose tracking was proposed in [3]. The kinematics of this configuration is studied, for example, in [5] and [6]. Its direct kinematics can be solved in closed form by using three consecutive trilateration operations yielding eight solutions [7]. The 2-2-2 configuration was first proposed in [4] for a wire-based tracking device. The kinematics of this configuration is studied, for example, in [8]-[10], where it is shown that its forward kinematics has 16 solutions. In other words, there are up to 16 poses for the moving object compatible with a given set of wire lengths. These configurations can only be obtained by numerical methods. The aforementioned two configurations were compared in terms of their sensitivity to wire-length errors in [3]. The conclusion was that they have similar properties.

In order to reduce cable interferences, singularities, and wire tension problems, we choose to reduce the number of cables from six to three, and to have the base rotate on its center (see Fig. 1). Provided that the tracked object motion is sufficiently slow, two measurements at different base orientations would be equivalent to a 2-2-2 configuration. More elegantly, and to let 


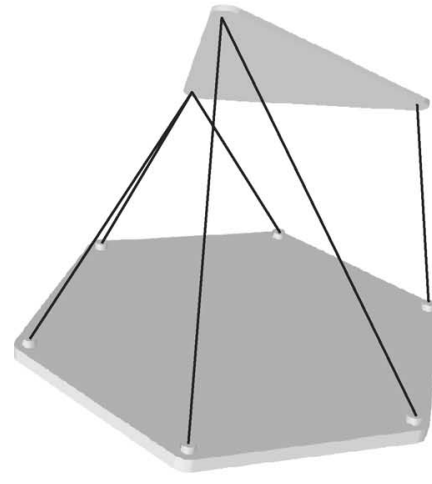

(a)

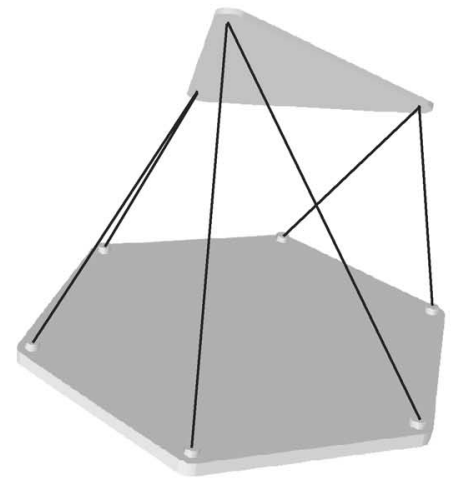

(b)

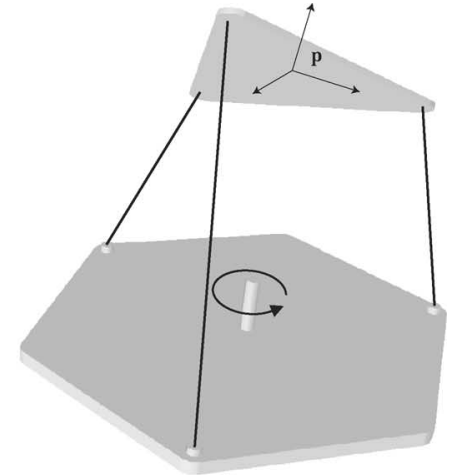

(c)

Fig. 1. Different configurations of wire-based tracking devices. (a) 3-2-1 configuration. (b) 2-2-2 configuration. (c) Proposed tracking device.

the tracked object move at faster speeds, measurements can be integrated sequentially through a partially observable estimation framework. Following the convention of naming the devices by the configuration of the attaching points on the moving object, the proposed three-wire tracker has a 1-1-1 configuration.

The paper is organized as follows. Section II contains the kinematics model of our proposed three-wire sensing device; Section III describes the filtering strategy for pose tracking. Given that this device has a moving part, Section IV develops an information theoretic metric for choosing the best actions for controlling it. A strategy to prevent possible wire crossings is contemplated in Section V. Section VI provides a workspace analysis and Section VII is devoted to a set of examples demonstrating the viability of the proposed device. Finally, concluding remarks are presented in Section VIII.

\section{Kinematics of the Proposed Sensor}

Consider the three-wire parallel device from Fig. 1(c). It is assumed that the platform is free to move in any direction in $\mathbb{R}^{3} \times \mathrm{SO}(3)$. Let the state of our tracking device be defined as the 12-dimensional array

$$
\mathrm{x}=\left[\begin{array}{c}
\mathrm{p} \\
\boldsymbol{\theta} \\
\mathrm{v} \\
\omega
\end{array}\right]
$$

where $\mathbf{p}=(x, y, z)^{\top}$ is the position of the origin of a coordinate frame fixed to the platform, $\boldsymbol{\theta}=(\psi, \theta, \phi)^{\top}$ is the orientation of such coordinate frame expressed as yaw, pitch, and roll angles, and $\mathbf{v}=\left(v_{x}, v_{y}, v_{z}\right)^{\top}$ and $\boldsymbol{\omega}=\left(\omega_{x}, \omega_{y}, \omega_{z}\right)^{\top}$ are the translational and rotational velocities of $\mathbf{p}$, respectively.

Assume that the attaching points on the base $\mathbf{a}_{i}, i=1,2,3$, are distributed on a circle of radius $a$ as shown in Fig. 2, with $\rho_{1}=0, \rho_{2}=2 \pi / 3$, and $\rho_{3}=4 \pi / 3$. Then, the coordinates of $\mathbf{a}_{i}$ can be expressed in terms of the base rotation angle $\beta$ as

$$
\left[\begin{array}{c}
a_{x i} \\
a_{y i} \\
a_{z i}
\end{array}\right]=\left[\begin{array}{c}
a \cos \left(\beta+\rho_{i}\right) \\
a \sin \left(\beta+\rho_{i}\right) \\
0
\end{array}\right] .
$$

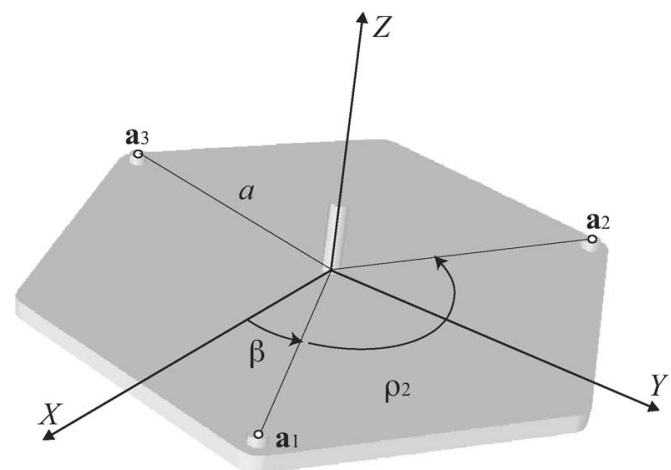

Fig. 2. Location of the wire attaching points in the rotating base.

Moreover, let $\mathbf{e}_{i}$ be the unit vector specifying the direction from $\mathbf{a}_{i}$ to the corresponding attaching point $\mathbf{b}_{i}$ in the platform, and let $l_{i}$ be the length of the $i$ th wire. The position of the wire attaching points in the platform in global coordinates are

$$
\mathbf{b}_{i}=\mathbf{a}_{i}+l_{i} \mathbf{e}_{i}=\mathbf{p}+\mathbf{R} \mathbf{c}_{i} .
$$

The vector $\mathbf{c}_{i}$ connects $\mathbf{p}$ to the attaching point $\mathbf{b}_{i}$. It is a known design constant and is expressed in platform local coordinates. $\mathbf{R}$ is the rotation matrix describing the orientation of the platform with respect to the base.

\section{STATE ESTIMATION}

The objective is to track the platform motion by measuring the length of the wires. We adopt a smooth unconstrained constant-velocity model for the motion of the platform, its pose altered only by zero-mean, normally distributed accelerations. This model is often used to track rigid bodies that move at or near constant velocity [11]. The Gaussian acceleration assumption means that large impulsive changes of direction are unlikely. In such model, the position and orientation of the platform and its translational and angular velocities, at time $t$ plus 
a time interval $\tau, \mathbf{x}(t+\tau)=\mathbf{f}\left(\mathbf{x}(t), \boldsymbol{\delta}_{x}(t)\right)$, are given by

$$
\left[\begin{array}{c}
\mathbf{p}(t+\tau) \\
\boldsymbol{\theta}(t+\tau) \\
\mathbf{v}(t+\tau) \\
\boldsymbol{\omega}(t+\tau)
\end{array}\right]=\left[\begin{array}{c}
\mathbf{p}(t)+\mathbf{v}(t) \tau+\boldsymbol{\delta}_{a}(t) \tau^{2} / 2 \\
\boldsymbol{\theta}(t)+\boldsymbol{\omega}(t) \tau+\boldsymbol{\delta}_{\alpha}(t) \tau^{2} / 2 \\
\mathbf{v}(t)+\boldsymbol{\delta}_{a}(t) \tau \\
\boldsymbol{\omega}(t)+\boldsymbol{\delta}_{\alpha}(t) \tau
\end{array}\right]
$$

with $\boldsymbol{\delta}_{x}=\left(\boldsymbol{\delta}_{a}, \boldsymbol{\delta}_{\alpha}\right)$ zero mean white Gaussian translational and angular acceleration noises $\boldsymbol{\delta}_{x} \sim \mathcal{N}\left(\mathbf{0}, \boldsymbol{\Sigma}_{x}\right)$.

Solving from (3), we obtain the kinematic position closure constraints that relate platform pose to the length of the wires $\mathbf{z}(t)=\mathbf{h}\left(\boldsymbol{x}(t), \boldsymbol{\delta}_{z}(t)\right)$, which for each wire is

$$
z_{i}(t)=l_{i}(t)+\delta_{z_{i}}(t)=\left\|\mathbf{p}(t)+\mathbf{R}(t) \mathbf{c}_{i}-\mathbf{a}_{i}(t)\right\|+\delta_{z_{i}}(t) .
$$

In practice, measured wire lengths will be corrupted by some sources of uncertainty (i.e., wire tension variations) that we model as additive Gaussian noise $\delta_{z_{i}} \sim \mathcal{N}\left(0, \sigma_{z}^{2}\right)$.

An extended Kalman filter can be used to propagate the platform pose and velocity estimates, and then, to refine these estimates through wire length measurements. The plant Jacobians with respect to the state and to the noise are the $12 \times 12$ constant matrix

$$
\mathbf{F}=\frac{\partial \mathbf{f}}{\partial \mathbf{x}}=\left[\begin{array}{cc}
\mathbf{I} & \tau \mathbf{I} \\
\mathbf{0} & \mathbf{I}
\end{array}\right]
$$

and the $12 \times 6$ constant matrix

$$
\mathbf{G}=\frac{\partial \mathbf{f}}{\partial \boldsymbol{\delta}_{x}}=\left[\begin{array}{c}
\frac{\tau^{2} \mathbf{I}}{2} \\
\tau \mathbf{I}
\end{array}\right] .
$$

The measurement Jacobian is the $3 \times 12$ matrix

$$
\mathbf{H}(t)=\left[\begin{array}{l}
\mathbf{H}_{1}(t) \\
\mathbf{H}_{2}(t) \\
\mathbf{H}_{3}(t)
\end{array}\right]
$$

where each row corresponds to each one of the three wires

$$
\mathbf{H}_{i}(t)=\frac{\partial \mathbf{h}_{i}}{\partial \mathbf{x}}=\left[\begin{array}{lll}
\mathbf{e}_{i}(t)^{\top} & \left(\mathbf{R c}_{i} \times \mathbf{e}_{i}(t)\right)^{\top} \mathbf{M} & \mathbf{0}_{1 \times 6}
\end{array}\right]
$$

with

$$
\mathbf{e}_{i}(t)=\frac{\mathbf{p}(t)+\mathbf{R}(t) \mathbf{c}_{i}-\mathbf{a}_{i}(t)}{\left\|\mathbf{p}(t)+\mathbf{R}(t) \mathbf{c}_{i}-\mathbf{a}_{i}(t)\right\|}
$$

and

$$
\mathbf{M}=\left[\begin{array}{ccc}
\cos \phi \cos \theta & -\sin \phi & 0 \\
\sin \phi \cos \theta & \cos \phi & 0 \\
-\sin \theta & 0 & 1
\end{array}\right]
$$

for which $\boldsymbol{\omega}=\mathbf{M} \dot{\boldsymbol{\theta}}$ is the total angular velocity derived from the Euler angular velocities.

For the sake of clarity, in the sequel, and when needed, time dependencies $t+\tau \mid t$ will be used to indicate prior estimates (before measurements are incorporated), and the terms $t \mid t$ and $t+\tau \mid t+\tau$ will represent posterior estimates (once measurements are taken into account). The prediction of the state and state covariance are given by

$$
\begin{aligned}
\mathbf{x}(t+\tau \mid t) & =\mathbf{f}(\mathbf{x}(t \mid t), \mathbf{0}) \\
\boldsymbol{\Sigma}(t+\tau \mid t) & =\mathbf{F} \boldsymbol{\Sigma}(t \mid t) \mathbf{F}^{\top}+\mathbf{G} \boldsymbol{\Sigma}_{x} \mathbf{G}^{\top}
\end{aligned}
$$

and the revision of the state estimate and state covariance are

$$
\begin{aligned}
\mathbf{x}(t+\tau, t+\tau)= & \mathbf{x}(t+\tau \mid t)+\mathbf{K}(\mathbf{z}(t+\tau) \\
& -\mathbf{h}(\mathbf{x}(t+\tau \mid t), \mathbf{0})) \\
\mathbf{\Sigma}(t+\tau \mid t+\tau)= & (\mathbf{I}-\mathbf{K H}) \mathbf{\Sigma}(t+\tau \mid t)
\end{aligned}
$$

with all instances of the Jacobian $\mathbf{H}$ evaluated at $t+\tau \mid t, \mathbf{K}=$ $\boldsymbol{\Sigma}(t+\tau \mid t) \mathbf{H}^{\top} \mathbf{S}^{-1}$, the usual Kalman gain, and $\mathbf{S}=\mathbf{H} \boldsymbol{\Sigma}(t+$ $\tau \mid t) \mathbf{H}^{\top}+\boldsymbol{\Sigma}_{z}$, the innovation covariance matrix where $\boldsymbol{\Sigma}_{z}=$ $\sigma_{z}^{2} \mathbf{I}_{3 \times 3}$.

Given that our estimation scheme is partially observable, wire length measurements can be used to revise state estimates along a three-dimensional subspace of the state space only. The information gained from measuring only three wires at a time is $\mathbf{H}^{\top} \mathbf{S}^{-1} \mathbf{H}$, and it is singular. The unobservable directions in state space are indicated by the null space of this matrix, whereas the directions in state space for which uncertainty can be reduced from these measurements are orthogonal to that null space. The control strategy described next will allow us to choose the best base rotation commands for reducing overall platform pose uncertainty from partial measurements.

\section{CONTROL StRategy}

In this section, we develop a control strategy for rotating the base of our proposed three-wire sensing device. The aim is to rotate the base in the direction that most reduces the uncertainty in the entire pose state estimate by using the information that should be gained from future wire measurements where such a move be made.

The essential idea is to use mutual information as a measure of the statistical dependence between the platform pose and the wire lengths. The mutual information is the relative entropy between the marginal density $p(\mathbf{x})$ and the conditional $p(\mathbf{x} \mid \mathbf{z})$

$$
\begin{aligned}
& \mathcal{I}(\mathbf{x}, \mathbf{z})=\mathcal{H}(\mathbf{x})-\mathcal{H}(\mathbf{x} \mid \mathbf{z}) \\
& \mathcal{I}(\mathbf{x}, \mathbf{z})=\int_{\mathbf{x}, \mathbf{z}} p(\mathbf{x}, \mathbf{z}) \log \frac{p(\mathbf{x} \mid \mathbf{z})}{p(\mathbf{x})} d \mathbf{x} d \mathbf{z} .
\end{aligned}
$$

Given that our variables of interest are multivariate Gaussian distributions, the parameters of the marginal density $p(\mathbf{x})$ are trivially the Kalman prior mean $\mathbf{x}(t+\tau \mid t)$ and covariance $\boldsymbol{\Sigma}(t+\tau \mid t)$. Moreover, the parameters of the conditional density $p(\mathbf{x} \mid \mathbf{z})$ precisely come from the Kalman update equations $\mathbf{x}(t+\tau \mid t+\tau)$ and $\boldsymbol{\Sigma}(t+\tau \mid t+\tau)$. Substituting the general form of the Gaussian distribution in (17), we obtain the expression

$$
\mathcal{I}(\mathbf{x}, \mathbf{z})=\frac{1}{2}(\log |\boldsymbol{\Sigma}(t+\tau \mid t)|-\log |\boldsymbol{\Sigma}(t+\tau \mid t+\tau)|) .
$$

Thus, in choosing a maximally mutually informative motion command, we are maximizing the difference between prior and posterior entropies [12], [13]. In other words, we are choosing the motion command that most reduces the uncertainty of $\mathbf{x}$ 


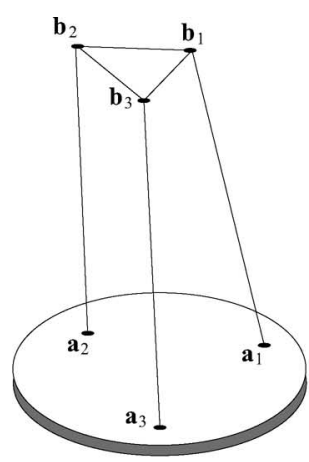

(a)

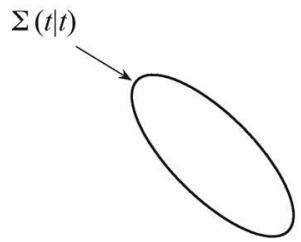

(d)

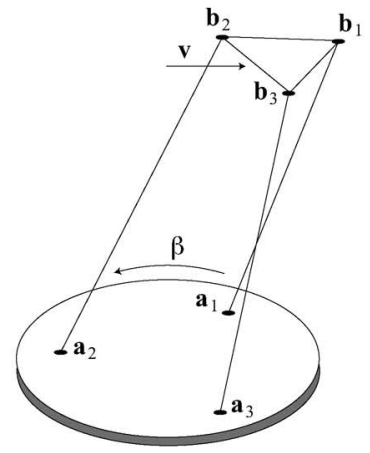

(b)

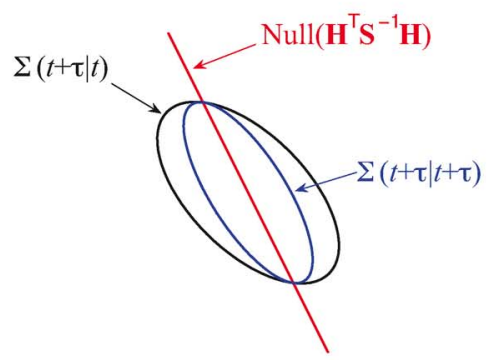

(e)

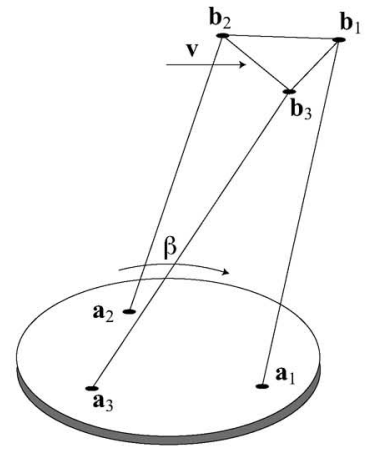

(c)

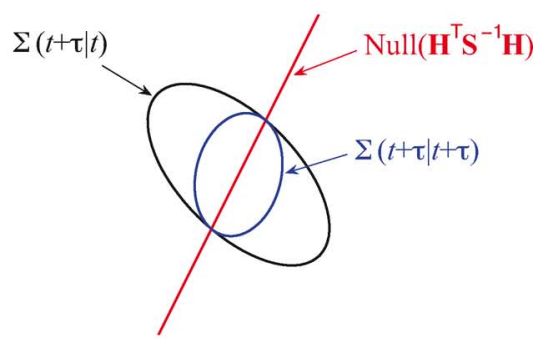

(f)

Fig. 3. Active sensing strategy. (a) Platform pose estimate at time $t$. (b) Platform moves at constant velocity v and the base rotates counterclockwise. (c) Same platform motion, but now the base rotates clockwise. (d) Hyperellipsoid of equal probability representing the covariance estimate at time $t$. (e) and (f) Predicted covariance increase $\boldsymbol{\Sigma}(t+\tau \mid t)$, unobservable directions in state space from the degenerate wire contribution $\mathbf{H}^{\top} \mathbf{S}^{-1} \mathbf{H}$ and revised covariance $\boldsymbol{\Sigma}(t+\tau \mid t+\tau)$ after the Kalman update.

due to the knowledge of $\mathbf{z}$. Observe, however, that given our constant velocity model for the prior estimation of platform pose, base orientation changes will not alter the prior covariance $\boldsymbol{\Sigma}(t+\tau \mid t)$. The orientation change $\beta$ affects only the location of the attaching points in the base $\mathbf{a}_{i}$ and cannot modify the constant Jacobians $\mathbf{F}$ or $\mathbf{G}$.

For this reason, for our particular system, when comparing the gain in mutual information induced by a set of motion commands, we only need to analyze the changes in posterior entropy [second term in (18)]

$$
\mathcal{H}(\mathbf{x} \mid \mathbf{z})=\frac{1}{2} \log |\boldsymbol{\Sigma}(t+\tau \mid t+\tau)|
$$

and choose the action that minimizes it:

$$
\beta^{*}=\underset{\beta \in U}{\operatorname{argmin}}|\boldsymbol{\Sigma}(t+\tau \mid t+\tau)| .
$$

The real-time requirements of the task preclude evaluating such metric for a large discrete set of actions within the range of possible commands. In our case, the set of actions evaluated at fixed values of $\beta$ are $U=$ \{rotate-left, stop, rotate-right $\}$. The scheme is illustrated in Fig. 3. Fig. 3(a) exemplifies one possible platform pose. The platform is expected to move at constant velocity, indicated by the vector $\mathbf{v}$. Two choices for rotating the base need be analyzed, counterclockwise [Fig. 3(b)] and clockwise [Fig. 3(c)]. During the Kalman prediction step, each base rotation command would induce an expected growth in the estimated platform position uncertainty, from the covariance $\boldsymbol{\Sigma}(t \mid t)$ shown in Fig. 3(d) to the larger covariance $\boldsymbol{\Sigma}(t+\tau \mid t)$ in Fig. 3(e) and (f). During the Kalman update, expected measurements from the two different wire configurations would reduce the estimated platform position uncertainty by different amounts, depending on the direction of the degenerate contribution $\mathbf{H}^{\top} \mathbf{S}^{-1} \mathbf{H}$. The command chosen is the one for which the posterior entropy is minimized, and in this particular case, that of Fig. 3(c) and (f). Similar applications requiring real-time performance that use mutual information to base their actions have been reported in [12] and [14].

\section{Preventing Wire Crossings}

Providing the base with the ability to rotate has the added advantage of increasing the range of motion of the tracked platform, mainly for rotations along the vertical axis. Special care must be taken however, to ensure that such base rotations will not induce wire crossings. Considering that wire end-point displacements are sufficiently small per sampling interval, and that the platform moves at constant velocity, the trajectory described by each wire can be assumed to be bounded by the tetrahedron described by the prior and posterior estimates of the wire attaching points at the base and the platform (see Fig. 4). One way to guarantee that such wire crossings will not occur is by checking whether these tetrahedra do not intersect each other. That is, we must verify that for the three wires, the tetrahedra described by the four attaching points $\left\{\mathbf{a}_{i}(t \mid t), \mathbf{a}_{i}(t+\tau \mid t), \mathbf{b}_{i}(t \mid t), \mathbf{b}_{i}(t+\tau \mid t)\right\}$ do not intersect.

A very fast test for tetrahedra intersection is based on the separating axis theorem described in the computer graphics literature [15]. The test consists of checking whether the plane lying on the face of each tetrahedra separates the two of them. 


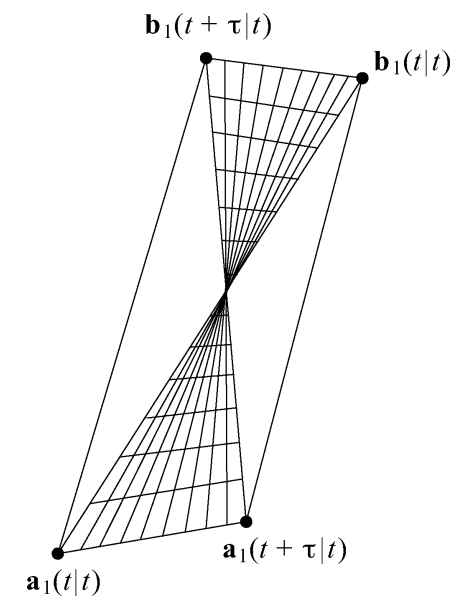

Fig. 4. Tetrahedron defined by the prior and posterior estimates of the wire attaching points at the base, and the platform is a convex bound of the trajectory of such wire moving at constant velocity.

To implement this test, we need to compare the signed volume described by the tetrahedron of one wire to the signed volume of the four tetrahedra formed by joining the candidate separating face with each of the vertices in the tetrahedron of the other wire. Fig. 5 shows two possible trajectories for wires 1 and 2. When the face under scrutiny is $\left\{\mathbf{a}_{2}(t \mid t), \mathbf{a}_{2}(t+\tau \mid t), \mathbf{b}_{2}(t \mid t)\right\}$ and the trajectories do not intersect [see Fig. 5(a)], the following must hold

$$
\begin{aligned}
& \mathbf{A}=\left[\begin{array}{lll}
\mathbf{a}_{2}(t \mid t) & \mathbf{a}_{2}(t+\tau \mid t) & \mathbf{b}_{2}(t \mid t)
\end{array}\right] \\
& \operatorname{sgn}\left|\begin{array}{cc}
\mathbf{A} & \mathbf{a}_{1}(t \mid t) \\
\mathbf{1}_{1 \times 3} & 1
\end{array}\right|=\operatorname{sgn}\left|\begin{array}{cc}
\mathbf{A} & \mathbf{a}_{1}(t+\tau \mid t) \\
\mathbf{1}_{1 \times 3} & 1
\end{array}\right| \\
& =\operatorname{sgn}\left|\begin{array}{cc}
\mathbf{A} & \mathbf{b}_{1}(t \mid t) \\
\mathbf{1}_{1 \times 3} & 1
\end{array}\right|=\operatorname{sgn}\left|\begin{array}{cc}
\mathbf{A} & \mathbf{b}_{1}(t+\tau \mid t) \\
\mathbf{1}_{1 \times 3} & 1
\end{array}\right| \\
& \neq \operatorname{sgn}\left|\begin{array}{cc}
\mathbf{A} & \mathbf{b}_{2}(t+\tau \mid t) \\
\mathbf{1}_{1 \times 3} & 1
\end{array}\right| \text {. }
\end{aligned}
$$

However, when the plane on which the face $\left\{\mathbf{a}_{2}(t \mid t), \mathbf{a}_{2}(t+\right.$ $\left.\tau \mid t), \mathbf{b}_{2}(t \mid t)\right\}$ lies is not a separating one [see Fig. 5(b)], then at least one of the four vertices in the last column of the determinants on the left-hand side of the inequality will produce a signed volume with the same sign as the one for $\mathbf{b}_{2}(t+\tau \mid t)$ on the right-hand side of the inequality. In that case, the test must be exhausted for the two remaining faces of the tetrahedron. If the search for a separating plane completes without success, then we can say that the proposed wire trajectories may intersect and the causing motion command must be avoided.

\section{WORKSPACE ANALYSIS}

In this section, we present an algorithm to compute the total orientation workspace (TOW) of our device and compare it with that of a 3-2-1 parallel device of similar dimensions. The workspace of these parallel wire sensors is mainly limited by the minimal and maximal wire lengths, by the cone angles of

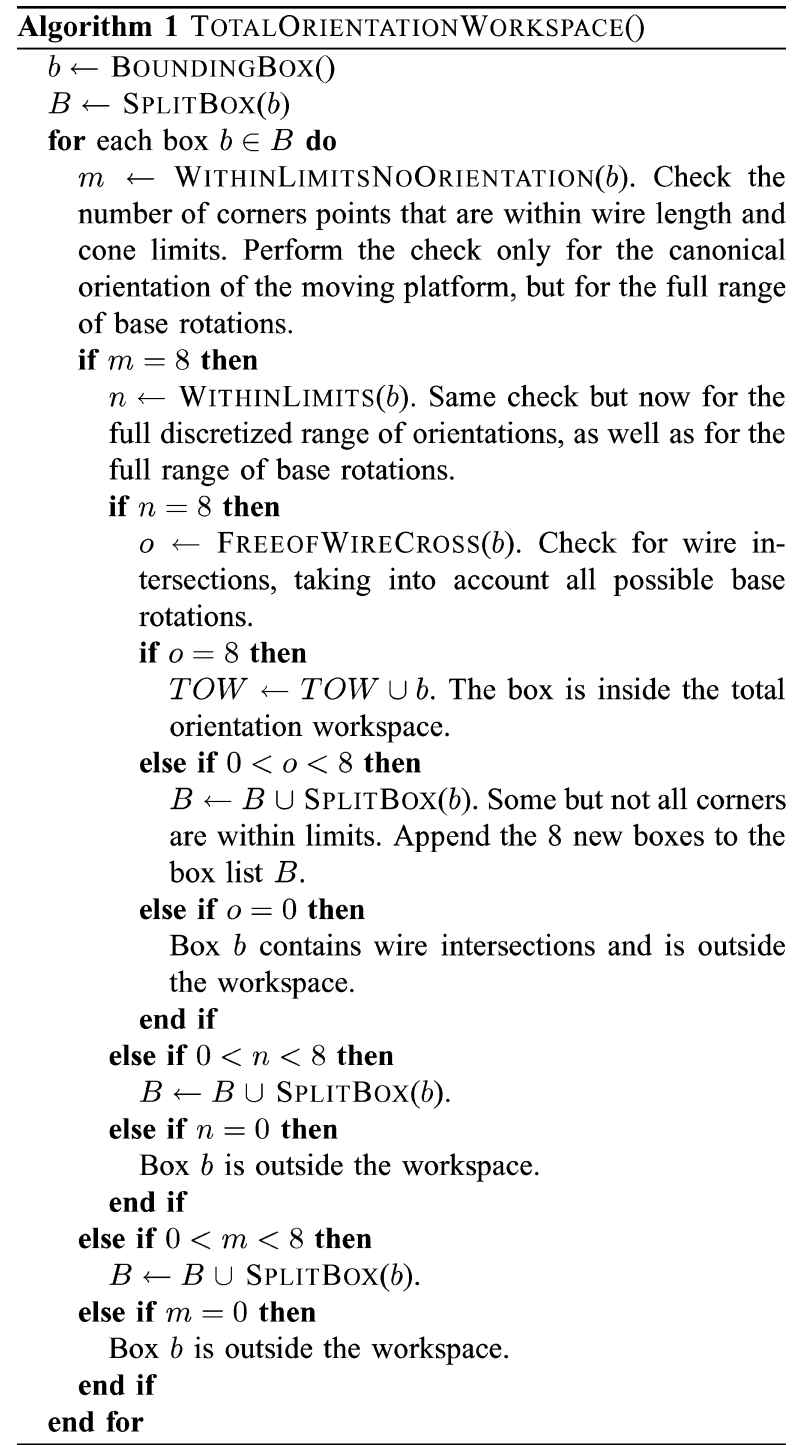

the wires at the base attaching points, and by wire interferences. Wire interference is by far the most critical of these limitations.

The TOW includes all possible locations of the center of the platform that can be reached with any orientation in a set defined by three ranges for the orientation angles. Our algorithm for computing the TOW is a simplification of the recursive algorithm proposed in [16] that includes a wire crossing test.

The algorithm starts by computing the workspace bounding box, defined as the intersection of the bounding box for each wire, a half cube whose base is centered in $\mathbf{a}_{i}$, their base edges have length $2\left(l_{i, \max }+\left\|\mathbf{c}_{i}\right\|\right)$, and has height $l_{i, \max }+\left\|\mathbf{c}_{i}\right\|$. The algorithm then splits the bounding box into eight boxes, and for each of them, checks whether each corner can be reached by the platform with any orientation inside the desired range, and without wire intersection. If the test fails, the box is divided, and the test is recursively called on each of the smaller boxes until all corners of the box fall either inside or outside of the TOW, or until a box division smaller than an accuracy level $\varepsilon$ is reached.

Our algorithm, detailed in Algorithm 1, in contrast to the one presented in [16], also has the added complexity of 


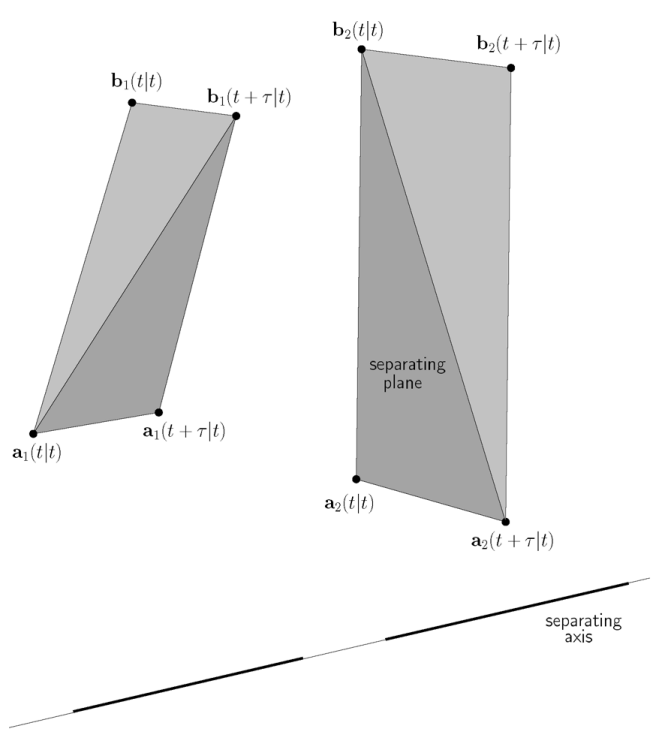

(a)
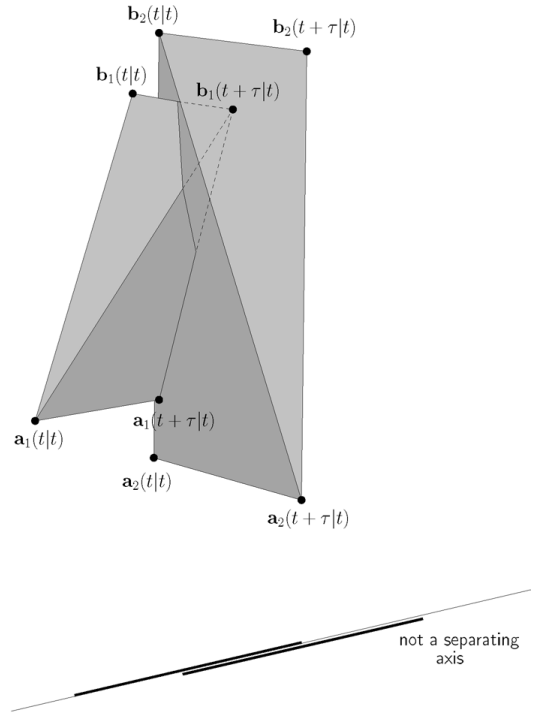

(b)

Fig. 5. To test for wire crossings, the signed volumes for the tetrahedra formed by grouping the separating plane and each vertex of one of the tetrahedra must be of opposite sign to the volume of the other tetrahedron. (a) Tetrahedra do not intersect. (b) Tetrahedra intersect.

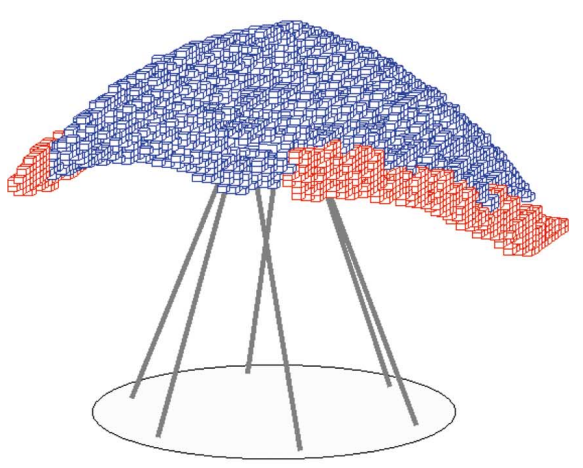

(a)

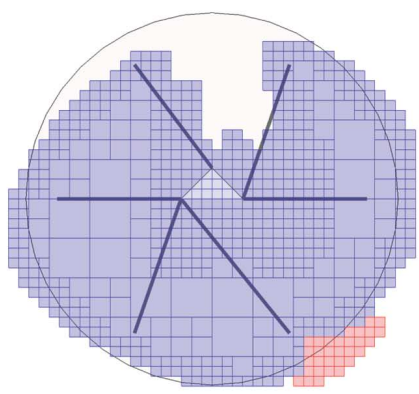

(c)

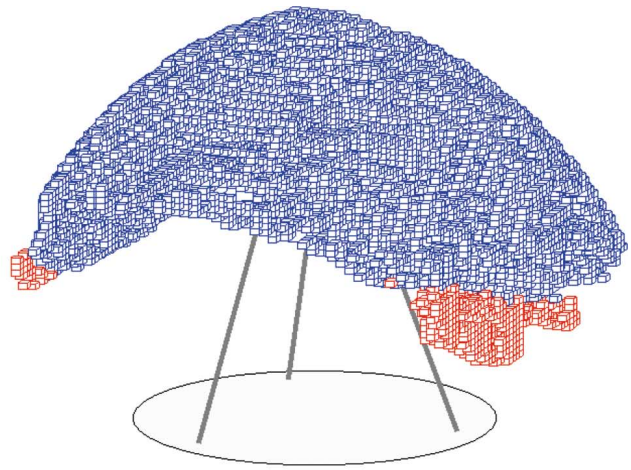

(b)

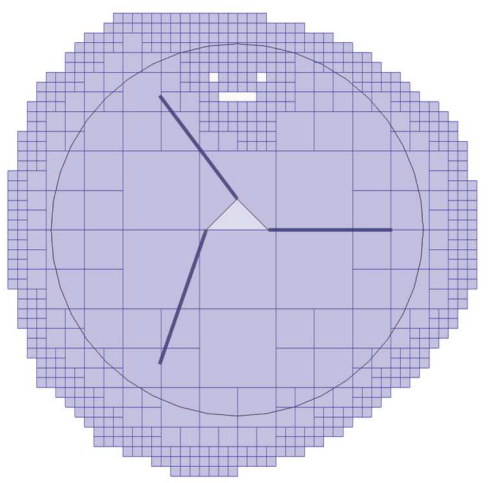

(d)

Fig. 6. Comparison of the TOWs for (a) 3-2-1 wire sensor. (b) Proposed 1-1-1 device. The cross sections (c) and (d) are coplanar with the canonical platform pose shown in (a) and (b) The light gray blocks (red in the online version of the paper) indicate wire crossings.

varying the base orientation when testing for valid sensor poses. It must be noted, however, that our algorithm does not make use of interval analysis and checks only for valid configurations at the vertices of the boxes, and for a discretized range of base orientations. These approximations, however, are sufficient in our opinion to show that the proposed device has a significantly larger workspace than its 3-2-1 sibling, which is the main purpose of this section.

Fig. 6 shows a comparison of the TOWs for a 3-2-1 sensor and for the proposed three-wire device of similar dimensions. 
TABLE I

TOW COMPUTATION PARAMETERS AND WORKSPACE SIZES

\begin{tabular}{lc}
\hline Parameter & Value \\
\hline Cable guiding cone & $40^{\circ}$ \\
Minimum wire length $l_{i, \min }$ & $0.5 \mathrm{~m}$ \\
Maximum wire length $l_{i, \text { max }}$ & $1.5 \mathrm{~m}$ \\
Box volume accuracy $\varepsilon$ & $(0.01 \mathrm{~m})^{3}$ \\
Orientation ranges (roll, pitch, and yaw) & {$[-\pi / 6, \pi / 6]$} \\
Orientation steps & 9 \\
Base rotation steps & 20 \\
Minimum distance between wires & $0.02 \mathrm{~m}^{3}$ \\
\hline 3-2-1 total orientation workspace & $0.3938 \mathrm{~m}^{3}$ \\
3-2-1 cross section area & $0.6784 \mathrm{~m}^{2}$ \\
1-1-1 total orientation workspace & $0.7246 \mathrm{~m}^{3}$ \\
1-1-1 cross section area & $1.3850 \mathrm{~m}^{2}$ \\
\hline
\end{tabular}

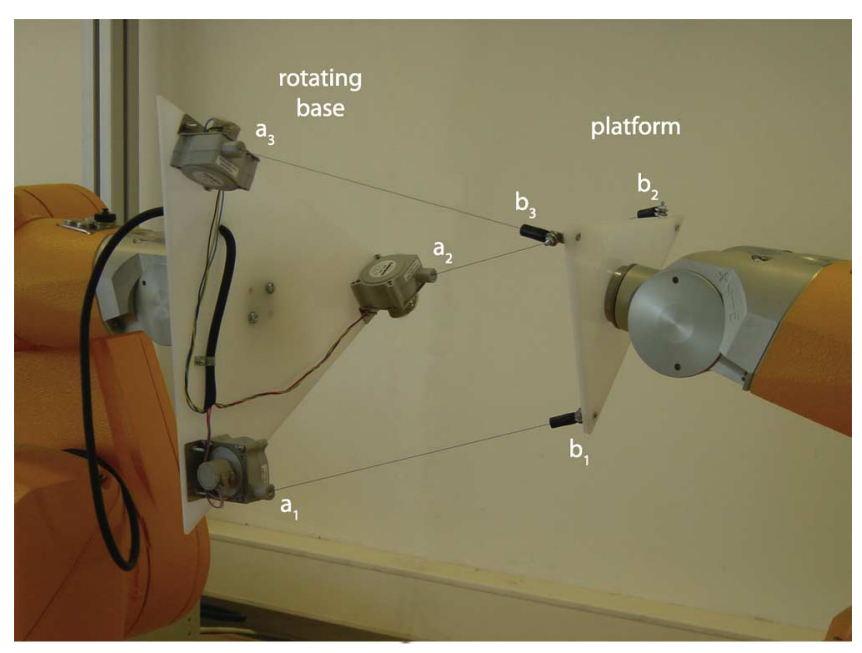

Fig. 7. A testbed for the proposed 3-wire active sensing device.

The parameters used in the computation of the workspaces and the resulting workspace sizes are summarized in Table I. The three-wire device has a workspace volume of almost double the size of its 3-2-1 counterpart. The cross section of the TOWs in Fig. 6(c) and (d) shows how the workspace of the 3-2-1 sensor is limited by the maximal wire lengths. In the plots, wire crossings are shown in red.

\section{IMPLEMENTATION AND EXAMPLES}

\section{A. Mechanical Considerations of the Implemented Test Bed}

The usefulness of a tracking device depends on whether it can track the motion fast enough. This ability is determined by the lag, or latency, between the change of the position and orientation of the target being tracked and the report of the change to the computer. In virtual reality applications, lags above $50 \mathrm{~ms}$ are perceptible to the user. The lag of mechanical trackers is typically less than $5 \mathrm{~ms}$.

The implemented system consists of a methacrylate triangular base with $0-1.27 \mathrm{~m} \mathrm{SP1}$ Celesco string pots attached to its corners, as shown in Fig. 7. The tension in the cable is guaranteed
TABLE II

COORDINATES OF THE ATTACHING POINTS (IN METERS) IN THEIR LOCAL COORDINATE FRAMES

\begin{tabular}{cccc}
\hline & $x$ & $y$ & $z$ \\
\hline $\mathbf{a}_{1}$ & 0.3000, & 0.3000, & -0.6000 \\
$\mathbf{a}_{2}$ & -0.1500, & 0.5598, & -0.6000 \\
$\mathbf{a}_{3}$ & -0.1500, & 0.0402, & -0.6000 \\
$\mathbf{b}_{1}$ & -0.0600, & 0.0000, & 0.0300 \\
$\mathbf{b}_{2}$ & 0.0600, & 0.0000, & 0.0300 \\
$\mathbf{b}_{3}$ & 0.0000, & 0.0600, & 0.0300 \\
\hline
\end{tabular}

by a spring connected to a string pot spool. These transducers have an accuracy of $0.025 \%$ of full scale.

The base is fixed on its center to a RX60 Staubli arm that induces rotation at desired values. The moving platform is also made of a triangular methacrylate piece holding the string pot end point attachments. This piece is mounted on a second Staubli arm to produce repeatable experiments in a measurable way. Ground-truth pose measurements are computed via the forward kinematics of the second Staubli arm. Wire length measurements are read on a PC by an off-the-shelf National Instruments Data Acquisition Card. To accommodate for unexpected target dynamics, the standard deviation in the plant model are $40 \mathrm{~mm} / \mathrm{s}^{2}$ and $0.14 \mathrm{rad} / \mathrm{s}^{2}$. The string pot used had a length measurement variance of $0.317 \mathrm{~mm}$.

The coordinates of the attaching points in both the base and the platform can be found in Table II, and refer to the frames shown in Fig. 1. In order to compare the performance of our sensor with respect to a 3-2-1 device, the mechanical dimensions of our system are at a perfect $3 / 5$ scale of the sensor reported in [7].

\section{B. Maximum Base Rotation Speed}

The quality of the estimated pose is directly influenced by the velocity at which the base can rotate. To determine the range of motion velocities that can be tracked with our system, a tracking simulation was repeated, limiting the base rotation velocity. A set of 20 runs was conducted, varying the maximum platform rotation speed from 0 to $\pi / 3 \mathrm{rad} / \mathrm{s}$, and with time steps of $0.01 \mathrm{~s}$, the tracked object translating at a constant velocity of $0.2 \mathrm{~m} / \mathrm{s}$ along the $x$-axis and rotating at $\pi / 10 \mathrm{rad} / \mathrm{s}$ about an axis perpendicular to the base. The best pose estimations are achieved when the base rotates at twice the speed of the tracked object, approximately $\pi / 5 \mathrm{rad} / \mathrm{s}$ for this experiment.

\section{Traversing a Singularity}

This experiment consists of approaching the sensor platform to the base plane while it is kept parallel to the $x$-axis of the base frame. Two base rotation strategies were used to track the platform. First, the platform was moved and stopped at each time cycle to allow the base to rotate to three locations at each iteration. This effectively allowed us to take one measurement for wire 1 (at angle $5 \pi / 6$ ), two for wire 2 (at angles $\pi / 6$ and $3 \pi / 2$ ), and three for wire 3 (at angles $\pi / 2,7 \pi / 6$, and $11 \pi / 6$ ), 


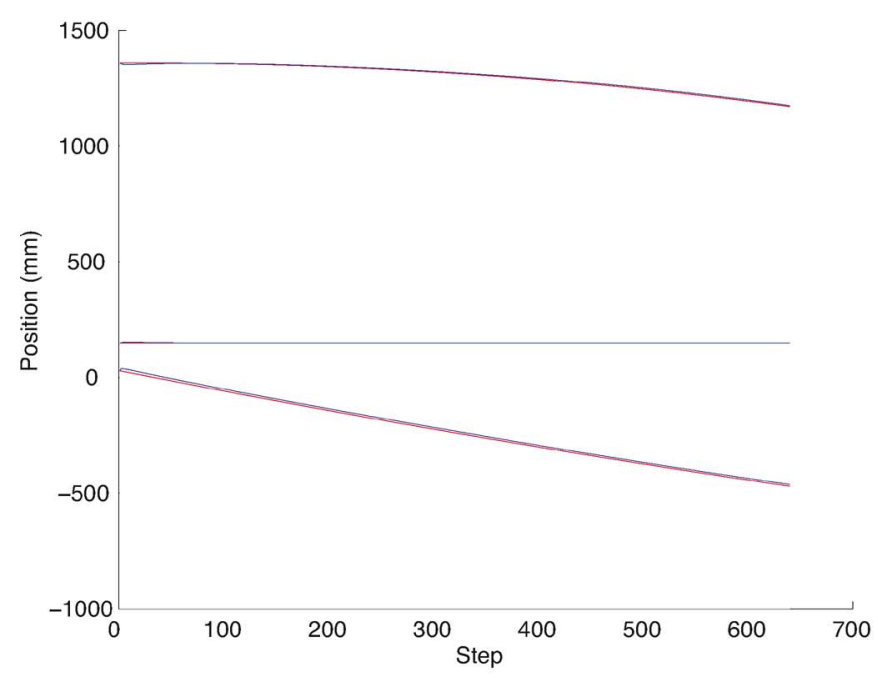

(a)

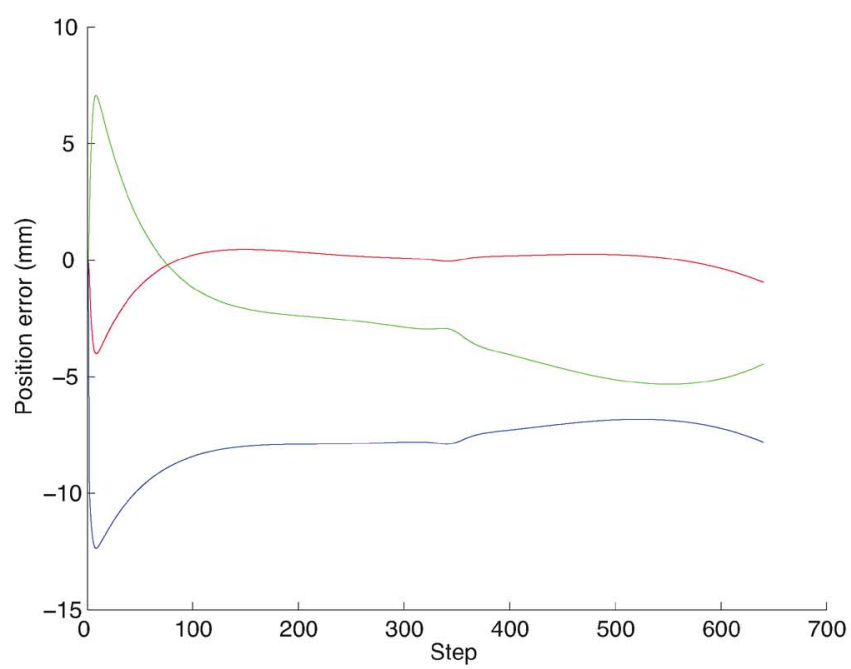

(c)

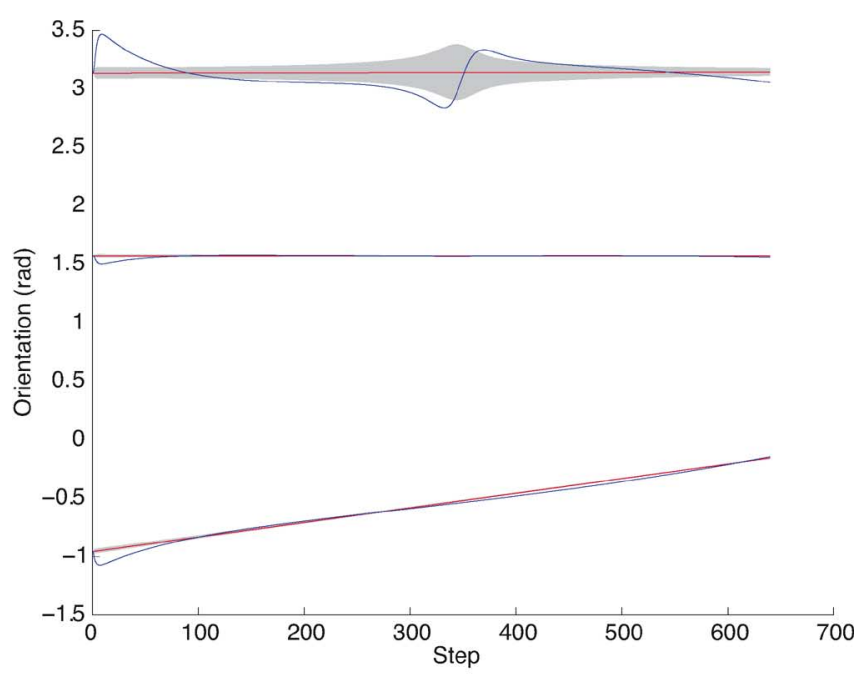

(b)

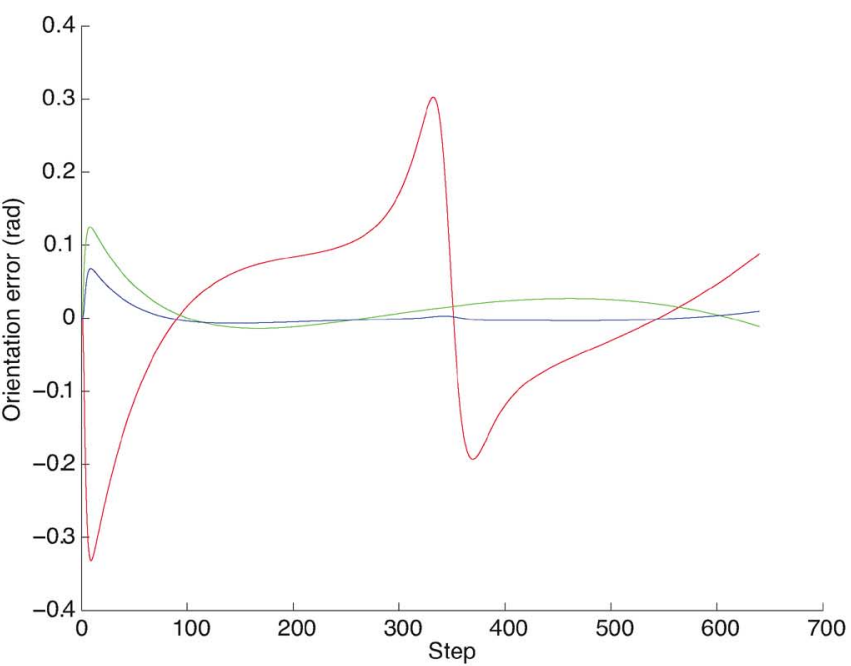

(d)

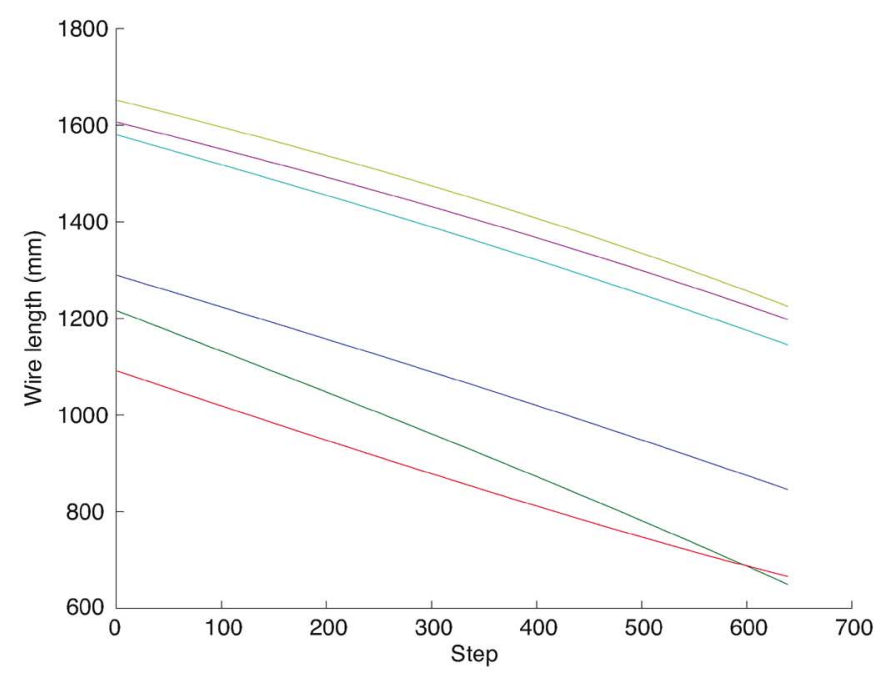

(e)

Fig. 8. Trajectory tracking of a 3-2-1 sensor when two solution branches intersect at a singularity. The shaded regions represent $2 \sigma$ confidence bounds for the estimated variances. 


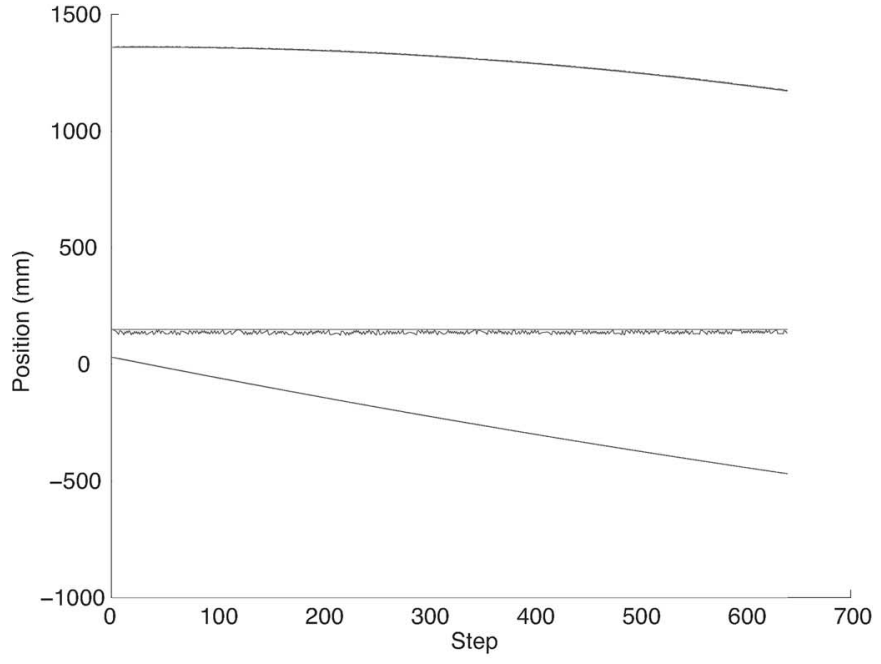

(a)

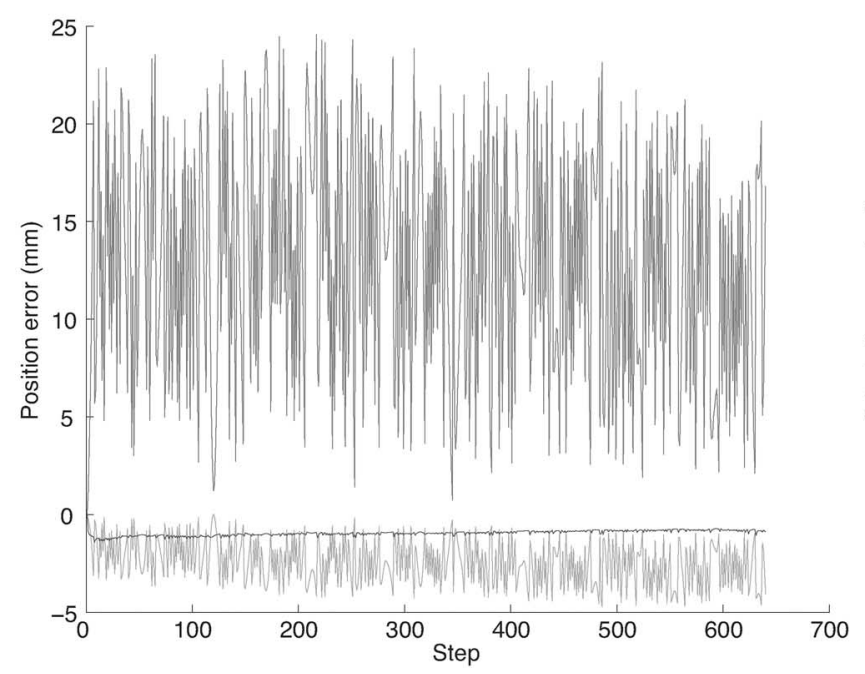

(c)

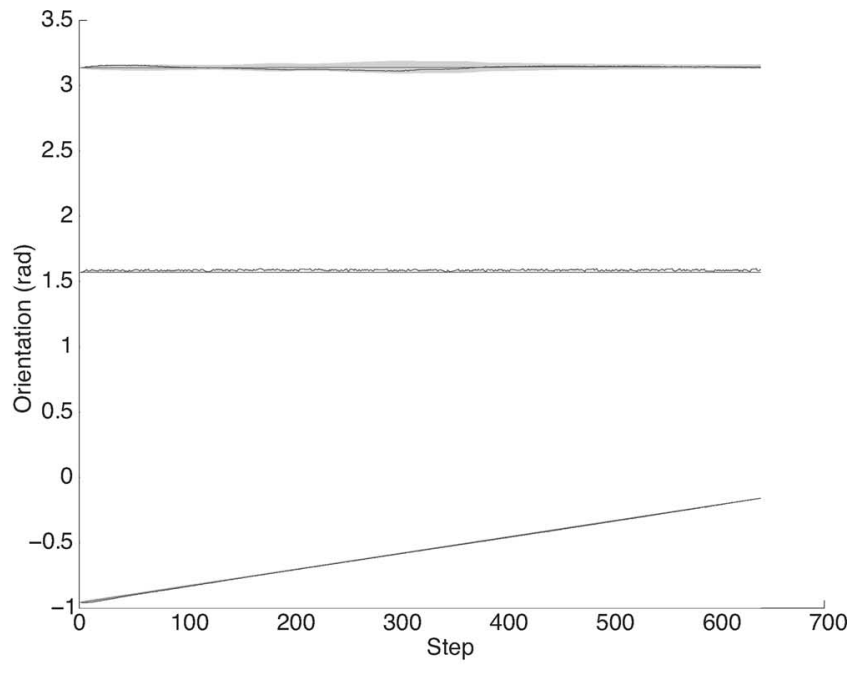

(b)

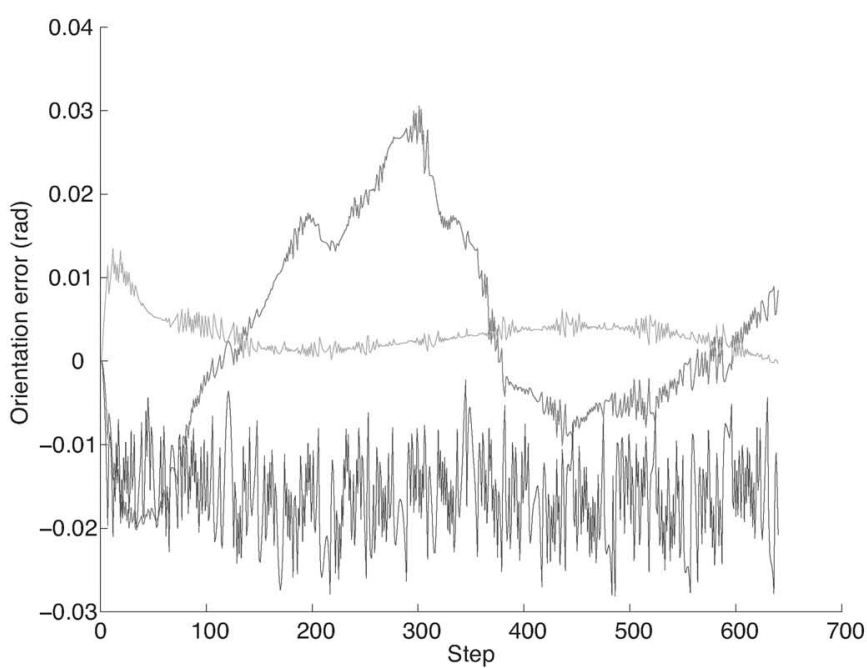

(d)

Fig. 9. Trajectory tracking for the proposed 1-1-1 device. The shaded regions represent $2 \sigma$ confidence bounds for the estimated variances.

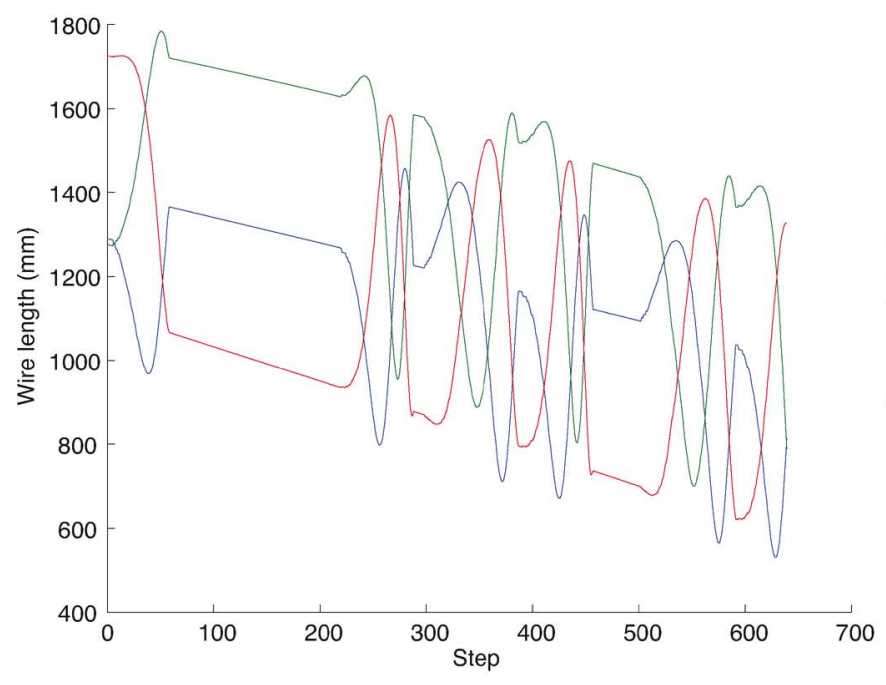

(a)

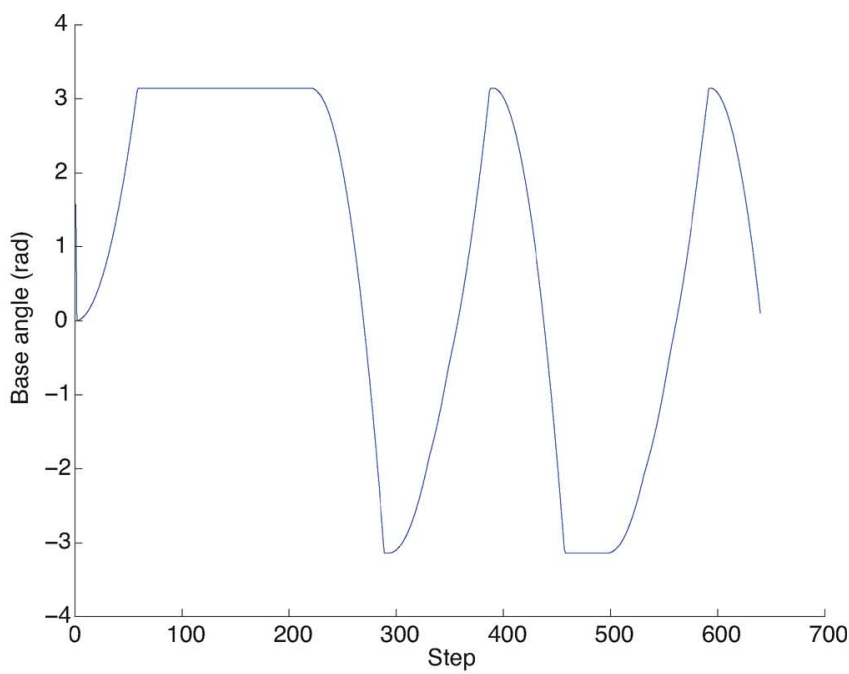

(b)

Fig. 10. Wire lengths and base angles for the proposed 1-1-1 device. 
while the platform was maintained still, thus replicating a 3-2-1 configuration. The trajectory chosen is of particular interest since it reaches a singular point near step 350, when the platform becomes coplanar with the plane $\left(\mathbf{b}_{1}, \mathbf{b}_{2}, \mathbf{a}_{3}\right)$. At that point, two solution branches for the forward kinematics of the 3-2-1 intersect and the uncertainty in pose estimate degenerates (see first experiment [7]). In theory, since infinite accelerations are not physically feasible, the continuity of the velocity vector for the motion model will predict the next pose near the real trajectory. Then, the filter might recover and follow the trajectory correctly (see Fig. 8). Unfortunately, due to the magnification of noise near a singularity, this cannot be guaranteed. In this experiment, to allow the system to recover from badly estimated rotations, the plant noise variance for rotations was inflated by a factor of 8 .

For the second base rotation strategy, the active control technique from Section IV was put to work. The platform does not stop at each time step, and the base is actively rotated. Active rotation of the base effectively avoids singular configurations in which the planes involved in trilateration become coplanar (see Fig. 9).

It might seem that the system is always nearly singular because the attaching points do not move very far between consecutive samples during base rotation. However, given that pose estimates are not the result of computing the forward kinematics with two measurements only, but the integration of the entire measurement history, singularity is avoided effectively. This fact is reinforced by a strategy to choose action commands on the basis of mutual information scores. Those commands are, from a given set of choices, the ones that drive the system away from ill-posed configurations. Also, given that the base is placed on a motor with absolute angular encoding, base rotation joint limits are not an issue. The only limitation on an angular base rotation are wire interferences that are effectively anticipated with the proposed wire crossing algorithm, and further validated at execution time with a minimal distance-between-wires test. Fig. 10 shows the actual wire lengths and the base rotation angles for the aforementioned experiment.

\section{CONCLUSION}

An active sensing strategy for a wire tracking device has been presented. It has been shown how, by allowing the sensor platform to rotate about its center, a wider range of motions can be tracked by reducing the number of wires needed from six to three, combined with a classic state estimation scheme. Platform rotation commands are chosen so as to maximize the mutual information between poses and measurements, which, in our case, turns out to be equivalent to choosing those actions that most reduce state estimation entropy. A heuristic to prevent wire wrappings is also considered.

The feasibility of developing such a device has been shown with an implementation using a dual Staubli-arm workbench. Our experiments suggest as a limitation of the approach that the base rotation speed should be at least twice compared to platform speed.

\section{REFERENCES}

[1] C. Melchiorri and G. Vassura, "Development and application of wireactuated haptic interfaces," J. Robot. Syst., vol. 18, no. 12, pp. 755-768, 2001.

[2] E. Ottaviano and M. Ceccarelli, "Numerical and experimental characterization of singularities of a six-wire parallel architecture," Robotica, vol. 25, no. 3, pp. 315-324, 2007.

[3] Z. J. Geng and L. S. Haynes, "A 3-2-1 kinematic configuration of a Stewart platform and its application to six degree of freedom pose measurements," Robot. Comput.-Integr. Manuf., vol. 11, no. 1, pp. 23-34, 1994.

[4] J. Jeong, S. Kim, and Y. Kwak, "Kinematics and workspace analysis of a parallel wire mechanism for measuring a robot pose," Mech. Mach. Theory, vol. 34, no. 6, pp. 825-841, 1999.

[5] P. Nanua and K. Waldron, "Direct kinematics solution of a special parallel robot structure," in Proc. 8th CISM-IFToMM Sym. Theory Practice Robot Manipulators, Warsaw, Poland, 1990, pp. 134-142.

[6] K. Hunt and E. Primrose, "Assembly configurations of some in-parallelactuated manipulators," Mech. Mach. Theory, vol. 28, no. 1, pp. 31-42, 1993.

[7] F. Thomas, E. Ottaviano, L. Ros, and M. Ceccarelli, "Performance analysis of a 3-2-1 pose estimation device," IEEE Trans. Robot., vol. 21, no. 3, pp. 288-297, Jun. 2005.

[8] M. Griffis and J. Duffy, "A forward displacement analysis of a class of Stewart platforms," J. Robot. Syst., vol. 6, no. 6, pp. 703-720, 1989.

[9] P. Nanua, K. Waldron, and V. Murthy, "Direct kinematic solution of a Stewart platform," IEEE Trans. Robot. Autom., vol. 6, no. 4, pp. 438444, Aug. 1990.

[10] V. Parenti-Castelli and C. Innocenti, "Direct displacement analysis for some classes of spatial parallel machanisms," in Proc. 8th CISMIFToMM Sym. Theory Practice Robot Manipulators, Warsaw, Poland, 1990, pp. 126-133.

[11] W. Blair and T. Bar-Shalom, "Tracking maneuvering targets with multiple sensors: Does more data always mean better estimates?," IEEE Trans. Aerosp. Electron. Syst., vol. 32, no. 1, pp. 450-456, Jan. 1996.

[12] A. Davison, "Active search for real-time vision," in Proc. IEEE Int. Conf. Comput. Vision, Beijing, China, Oct. 2005, pp. 66-73.

[13] D. J. C. MacKay, "Information based objective functions for active data selection," Neural Comput., vol. 4, no. 4, pp. 589-603, 1992.

[14] T. Vidal-Calleja, A. Davison, J. Andrade-Cetto, and D. Murray, "Active control for single camera SLAM," in Proc. IEEE Int. Conf. Robot. Autom., Orlando, FL, May 2006, pp. 1930-1936.

[15] F. Ganovelli, F. Ponchio, and C. Rocchini, "Fast tetrahedron-tetrahedron overlap algorithm," J. Graph. Tools, vol. 7, no. 2, pp. 17-26, 2002.

[16] J. Merlet, "Determination of $6 \mathrm{~d}$ workspaces of Gough-type parallel manipulator and comparison between different geometries," Int. J. Robot Res., vol. 18, no. 9, pp. 902-916, 1999.

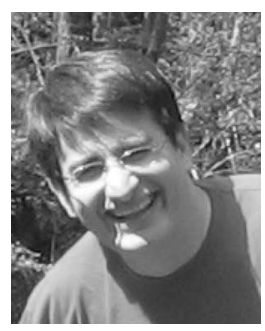

Juan Andrade-Cetto (S'94-M'95) received the B.S.E.E. degree from the Centro de Enseñanza Técnica y Superior (CETYS) Universidad, Baja California, Mexico, in 1993, the M.S.E.E. degree from Purdue University, West Lafayette, IN, in 1995 , and the Ph.D. degree in systems engineering from the Universitat Politècnica de Catalunya, Barcelona, Spain, in 2003.

$\mathrm{He}$ is currently a Researcher at the Spanish National Research Council, Institut de Robòtica i Informàtica Industrial, Barcelona. His current research interests include state estimation and computer vision with applications to mobile robotics problems.

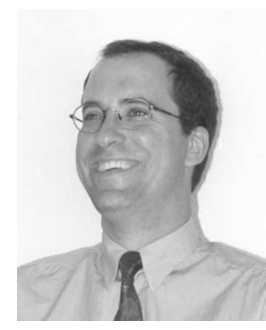

Federico Thomas (M'05) received the B.Sc. degree in telecommunications engineering and the Ph.D. (with honors) degree in computer science, both from the Universitat Politècnica de Catalunya, Barcelona, Spain, in 1984 and 1988, respectively.

He is currently a Professor of research at the Spanish National Research Council, Institut de Robòtica i Informàtica Industrial, Barcelona. His current research interests include geometry and kinematics, with applications to robotics, computer graphics, and machine vision.

Prof. Thomas is an Associate Editor of the IEEE TRANSACTIONS ON RoBOTICS and the Director of the Industrial Research Institute (IRI). 